\title{
Correction to: Measuring the persistence of high firm growth: choices and consequences
}

\section{Eva Christine Erhardt}

Published online: 15 January 2020

C) Springer Science+Business Media, LLC, part of Springer Nature 2020

\section{Correction to: Small Bus Econ}

https://doi.org/10.1007/s11187-019-00229-7

Unfortunately, the original version of this article was published online with error.

In equations (1) and (2) the variable 'epsilon' has disappeared. The corrected equation is shown below.

$$
\begin{aligned}
E\left(g_{i t+\tau} \mid H G F_{i t}, \boldsymbol{x}_{i t-3}\right)= & \beta_{0}+\beta_{1} H G F_{i t}+\boldsymbol{\beta}_{2} \boldsymbol{x}_{i t-3}+\varepsilon_{i t+\tau} \\
& \text { if survival } i t+\tau \\
E\left(g_{i t+\tau} \mid H G F_{i t}, \boldsymbol{x}_{i t-3}\right)= & \beta_{0}+\beta_{1} H G F_{i t}+\boldsymbol{\beta}_{2} \boldsymbol{x}_{i t-3}+\varepsilon_{i t+\tau} \\
& \text { if exit } \text { ext }_{i t \tau}
\end{aligned}
$$

The data in Tables 1, 3, 4, 5, 6, 13, 14, 15, 16, 17 and 18 were incorrectly displayed and aligned by the Springer proofreaders/or in the proofread stage of Springer.

The corrected Tables 1, 3, 4, 5, 6, 13, 14, 15, 16, 17 and 18 are shown in the next page.

Further, in the section Introdution, third line, the word 'fact' has been replaced with the word 'view'.

The original article has been corrected.

The online version of the original article can be found at https://doi.org/10.1007/s11187-019-00229-7

E. C. Erhardt $(\bowtie)$

Chair of Applied Statistics and Econometrics, University of Mainz, Jakob-Welder-Weg 4, 55128 Mainz, Germany

e-mail: erharde@uni-mainz.de 


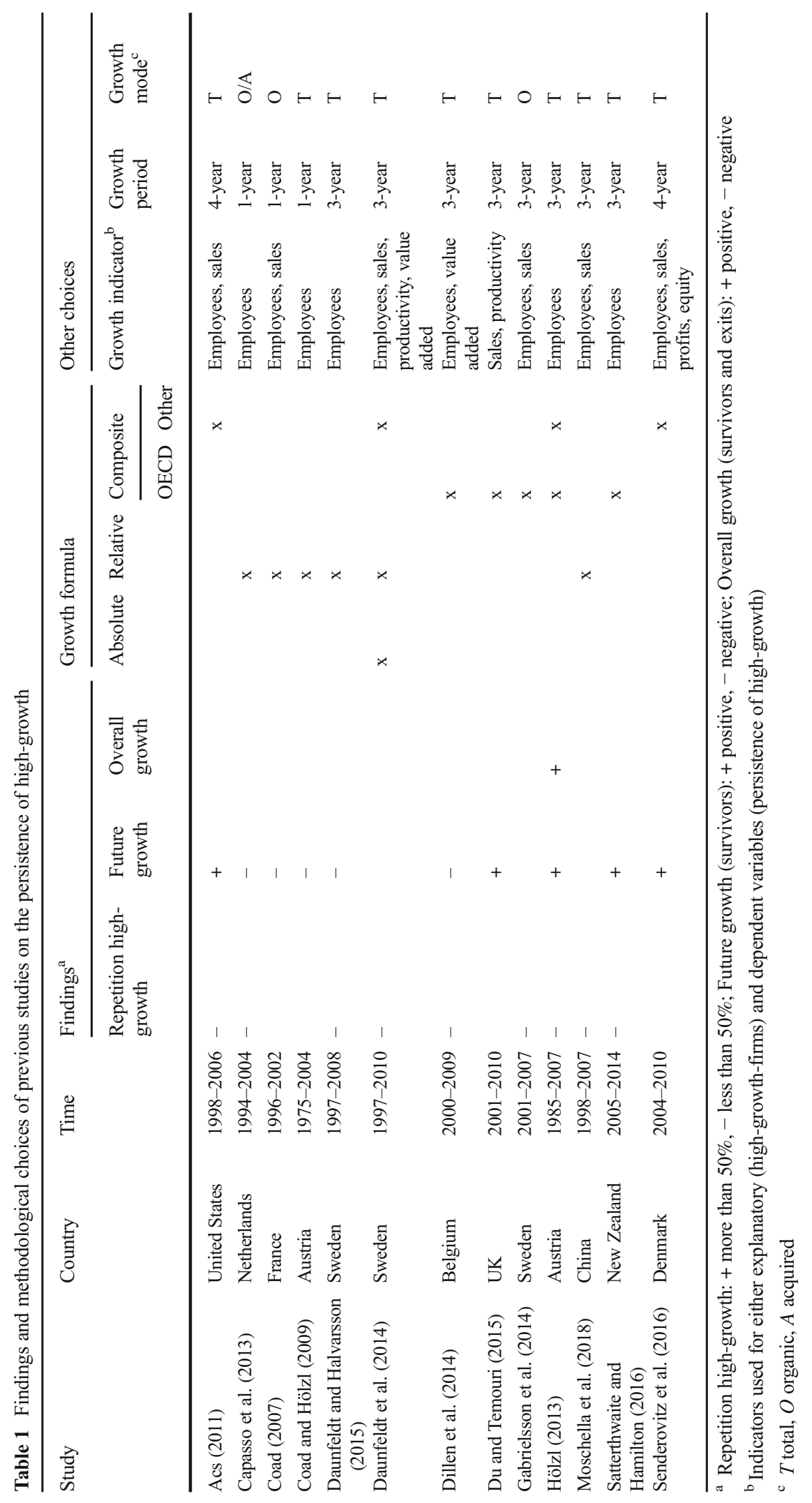


Table 3 Summary statistics of control variables

\begin{tabular}{|c|c|c|c|c|c|c|c|c|}
\hline \multirow{2}{*}{$\begin{array}{l}\text { Control variables } \\
\text { In mean (S.D.) }\end{array}$} & \multicolumn{2}{|c|}{ Absolute HGFs } & \multicolumn{2}{|c|}{ Relative HGFs } & \multicolumn{2}{|c|}{ OECD HGFs } & \multicolumn{2}{|c|}{ Total sample } \\
\hline & & & & & & & & \\
\hline Firm size in levels & 114.83 & (237.89) & 2.71 & $(6.73)$ & 33.60 & $(52.91)$ & 11.83 & (70.18) \\
\hline Age & 3.24 & $(2.25)$ & 1.91 & $(1.50)$ & 2.81 & $(1.90)$ & 2.49 & $(1.79)$ \\
\hline Industry size & 31.62 & $(59.10)$ & 15.55 & $(34.70)$ & 19.82 & (32.39) & 9.52 & $(22.49)$ \\
\hline \multicolumn{9}{|l|}{ Industry growth } \\
\hline Absolute & -6.72 & (20.17) & & & -0.08 & $(0.05)$ & -2.37 & $(7.62)$ \\
\hline Relative (percent) & & & -0.09 & $(0.05)$ & & & -0.09 & $(0.05)$ \\
\hline \multicolumn{9}{|l|}{ In share of total } \\
\hline \multicolumn{9}{|c|}{ Firm size in categories ${ }^{a}$} \\
\hline Micro $(<10)$ & 0.22 & & 0.96 & & - & & 0.82 & \\
\hline Small (10-49) & 0.31 & & 0.04 & & 0.85 & & 0.14 & \\
\hline Medium (50-250) & 0.36 & & - & & 0.14 & & 0.03 & \\
\hline Large $(>250)$ & 0.11 & & - & & 0.01 & & 0.01 & \\
\hline \multicolumn{9}{|l|}{ Industry code } \\
\hline Primary & 0.04 & & 0.04 & & 0.04 & & 0.03 & \\
\hline Secondary & 0.42 & & 0.29 & & 0.37 & & 0.19 & \\
\hline Tertiary & 0.55 & & 0.66 & & 0.60 & & 0.78 & \\
\hline Districts & yes & & yes & & yes & & yes & \\
\hline Legal form & yes & & yes & & yes & & yes & \\
\hline Observations & 3669 & & 3655 & & 5406 & & 369,294 & \\
\hline
\end{tabular}

Notes. Average values for periods 2001-2004, 2004-2007, and 2007-2010

${ }^{\text {a }}$ According to Eurostat

Table 4 Transition probabilities from $t$ to $t+3$, absolute HGFs

\begin{tabular}{llllll}
\hline & $g_{i, t+3}^{(0)}$ & $g_{i, t+3}^{(1)}$ & $g_{i, t+3}^{(2)}$ \\
negative growth & zero growth & $\begin{array}{l}g_{i, t+3}^{(3)} \\
\text { positive growth }\end{array}$ & $\begin{array}{l}g_{i, t+3}^{(4)} \\
\text { absolute HGF }\end{array}$ \\
\hline$g_{i, t}^{(1)}$ & 0.352 & 0.310 & 0.129 & 0.205 & 0.005 \\
$g_{i, t}^{(2)}$ & 0.452 & 0.086 & 0.353 & 0.109 & 0.001 \\
$g_{i, t}^{(3)}$ & 0.209 & 0.400 & 0.063 & 0.313 & 0.015 \\
$g_{i, t}^{(4)}=$ absolute HGF & 0.068 & 0.521 & 0.002 & 0.124 & 0.286 \\
\hline
\end{tabular}


Table 5 Transition probabilities from $t$ to $t+3$, relative HGFs

\begin{tabular}{|c|c|c|c|c|c|c|}
\hline & $\begin{array}{l}g_{i, t+3}^{(0)} \\
\text { exit }\end{array}$ & $\begin{array}{l}g_{i, t+3}^{(1)} \\
\text { negative growth }\end{array}$ & $\begin{array}{l}g_{i, t+3}^{(2)} \\
\text { zero growth }\end{array}$ & $\begin{array}{l}g_{i, t+3}^{(3)} \\
\text { positive growth }\end{array}$ & $\begin{array}{l}g_{i, t+3}^{(4)} \\
\text { relative HGF }\end{array}$ & Total \\
\hline$g_{i, t}^{(1)}$ & 0.352 & 0.310 & 0.129 & 0.204 & 0.005 & 1.000 \\
\hline$g_{i, t}^{(2)}$ & 0.452 & 0.086 & 0.353 & 0.106 & 0.004 & 1.000 \\
\hline$g_{i, t}^{(3)}$ & 0.203 & 0.404 & 0.062 & 0.323 & 0.004 & 1.000 \\
\hline$g_{i, t}^{(4)}=$ relative $\mathrm{HGF}$ & 0.271 & 0.376 & 0.021 & 0.325 & 0.007 & 1.000 \\
\hline
\end{tabular}

Table 6 Transition probabilities from $t$ to $t+3$, OECD HGFs

\begin{tabular}{|c|c|c|c|c|c|c|}
\hline & $\begin{array}{l}g_{i, t+3}^{(0)} \\
\text { exit }\end{array}$ & $\begin{array}{l}g_{i, t+3}^{(1)} \\
\text { negative growth }\end{array}$ & $\begin{array}{l}g_{i, t+3}^{(2)} \\
\text { zero growth }\end{array}$ & $\begin{array}{l}g_{i, t+3}^{(3)} \\
\text { positive growth }\end{array}$ & $\begin{array}{l}g_{i, t+3}^{(4)} \\
\text { OECD HGF }\end{array}$ & Total \\
\hline$g_{i, t}^{(1)}$ & 0.352 & 0.310 & 0.129 & 0.204 & 0.006 & 1.000 \\
\hline$g_{i, t}^{(2)}$ & 0.452 & 0.086 & 0.353 & 0.108 & 0.001 & 1.000 \\
\hline$g_{i, t}^{(3)}$ & 0.215 & 0.399 & 0.063 & 0.293 & 0.029 & 1.000 \\
\hline$g_{i, t}^{(4)}=\mathrm{OECD} \mathrm{HGF}$ & 0.078 & 0.464 & 0.018 & 0.326 & 0.115 & 1.000 \\
\hline
\end{tabular}

Transition probabilities are calculated using frequencies. Growth brackets are defined based on annualized percentage change in employees as follows: $g_{i, t+\tau}^{(0)}=-1$ (exit), $-1<g_{i, t+\tau}^{(1)}<0$ (negative growth), $g_{i, t+\tau}^{(2)}=0$ (zero growth), $g_{i, t+\tau}^{(3)}>0$ (positive growth), and $g_{i, t+\tau}^{(4)}=$ HGF. The definition of growth brackets based on absolute growth or log-differences does not alter results

Table 13 Transition probabilities from $t$ to $t+6$, absolute HGFs

\begin{tabular}{|c|c|c|c|c|c|}
\hline & $\begin{array}{l}g_{i, t+3}^{(0)} \\
\text { exit }\end{array}$ & $\begin{array}{l}g_{i, t+3}^{(1)} \\
\text { negative growth }\end{array}$ & $\begin{array}{l}g_{i, t+3}^{(2)} \\
\text { zero growth }\end{array}$ & $\begin{array}{l}g_{i, t+3}^{(3)} \\
\text { positive growth }\end{array}$ & $\begin{array}{l}g_{i, t+3}^{(4)} \\
\text { absolute HGF }\end{array}$ \\
\hline$g_{i, t}^{(1)}$ & 0.438 & 0.298 & 0.115 & 0.141 & 0.008 \\
\hline$g_{i, t}^{(2)}$ & 0.627 & 0.116 & 0.169 & 0.087 & 0.002 \\
\hline$g_{i, t}^{(3)}$ & 0.315 & 0.396 & 0.083 & 0.189 & 0.018 \\
\hline$g_{i, t}^{(4)}=$ absolute HGF & 0.098 & 0.697 & 0.010 & 0.052 & 0.142 \\
\hline
\end{tabular}


Table 14 Transition probabilities from $t$ to $t+6$, relative HGFs

\begin{tabular}{lllll}
\hline & $\begin{array}{l}g_{i, t+3}^{(0)} \\
\text { exit }\end{array}$ & $\begin{array}{l}g_{i, t+3}^{(1)} \\
\text { negative growth }\end{array}$ & $\begin{array}{l}g_{i, t+3}^{(2)} \\
\text { zero growth }\end{array}$ & $\begin{array}{l}g_{i, t+3}^{(3)} \\
\text { positive growth }\end{array}$ \\
\hline $\begin{array}{l}g_{i, t}^{(1)} \\
g_{i, t}^{(2)}\end{array}$ & 0.438 & 0.298 & 0.115 & 0.147 \\
$g_{i, t}^{(3)}$ & 0.627 & 0.116 & 0.169 & 0.087 \\
$g_{i, t}^{(4)}=$ relative HGF & 0.312 & 0.400 & 0.082 & 0.204 \\
\hline
\end{tabular}

Table 15 Transition probabilities from $t$ to $t+6$, OECD HGFs

\begin{tabular}{|c|c|c|c|c|c|}
\hline & $\begin{array}{l}g_{i, t+3}^{(0)} \\
\text { exit }\end{array}$ & $\begin{array}{l}g_{i, t+3}^{(1)} \\
\text { negative growth }\end{array}$ & $\begin{array}{l}g_{i, t+3}^{(2)} \\
\text { zero growth }\end{array}$ & $\begin{array}{l}g_{i, t+3}^{(3)} \\
\text { positive growth }\end{array}$ & $\begin{array}{l}g_{i, t+3}^{(4)} \\
\text { OECD HGF }\end{array}$ \\
\hline$g_{i, t}^{(1)}$ & 0.438 & 0.298 & 0.115 & 0.143 & 0.006 \\
\hline$g_{i, t}^{(2)}$ & 0.627 & 0.116 & 0.169 & 0.086 & 0.003 \\
\hline$g_{i, t}^{(3)}$ & 0.324 & 0.386 & 0.082 & 0.192 & 0.014 \\
\hline$g_{i, t}^{(4)}=\mathrm{OECD} \mathrm{HGF}$ & 0.127 & 0.622 & 0.028 & 0.199 & 0.024 \\
\hline
\end{tabular}

Transition probabilities are calculated using frequencies. Growth brackets are defined based on annualized percentage change in employees as follows: $g_{i, t+\tau}^{(0)}=-1$ (exit), $-1<g_{i, t+\tau}^{(1)}<0$ (negative growth), $g_{i, t+\tau}^{(2)}=0$ (zero growth), $g_{i, t+\tau}^{(3)}>0$ (positive growth), and $g_{i, t+\tau}^{(4)}=$ HGF. The definition of growth brackets based on absolute growth or log-differences does not alter results 


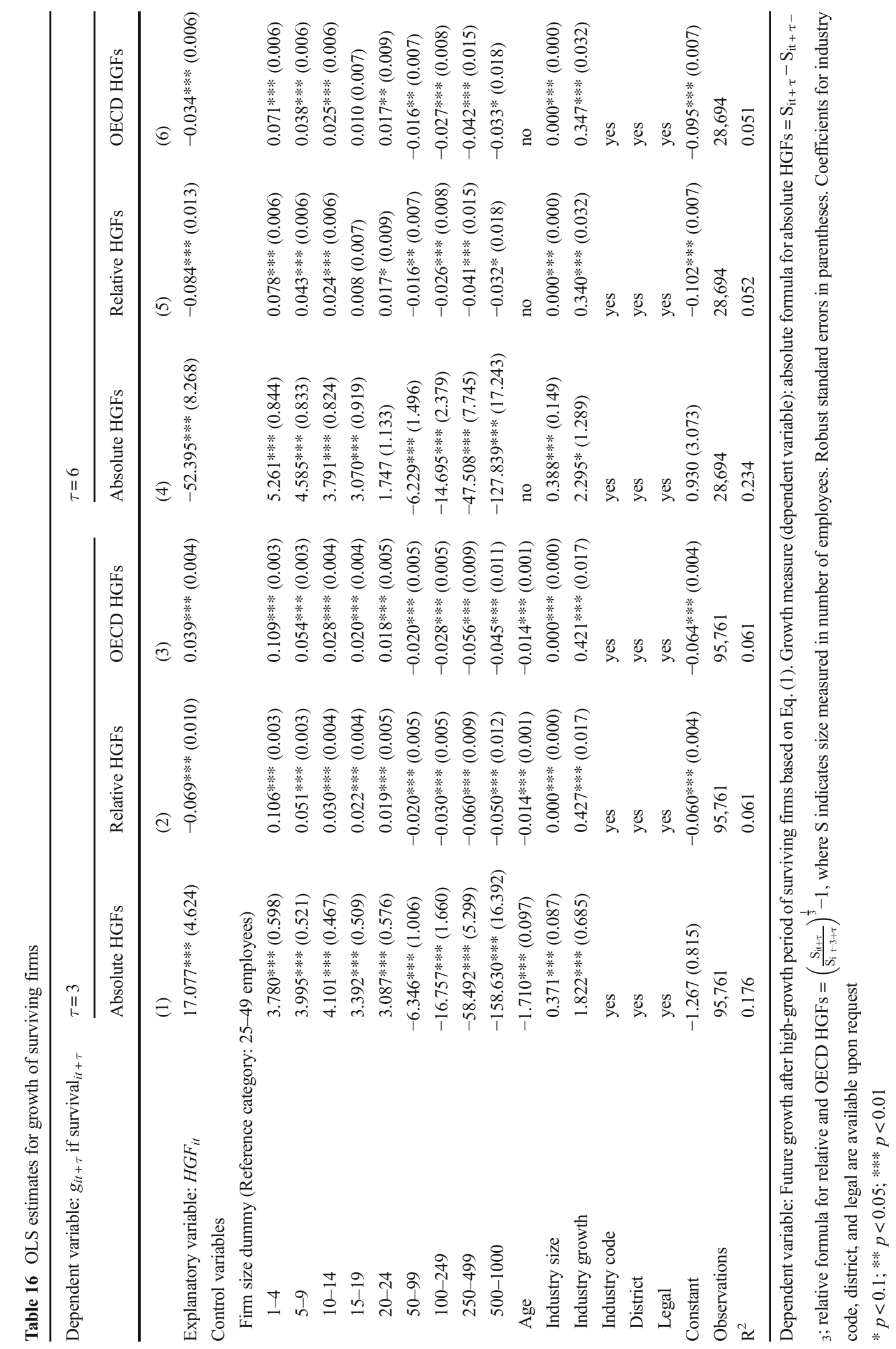


Table 17 OLS estimates for growth of exiting firms

\begin{tabular}{|c|c|c|}
\hline Dependent variable: $g_{i t+\tau}$ if $e x i t_{i t+\tau}$ & $\begin{array}{l}\tau=3 \\
\text { Absolute HGFs } \\
\text { (1) }\end{array}$ & $\begin{array}{l}\tau=6 \\
\text { Absolute HGFs } \\
\text { (2) }\end{array}$ \\
\hline Explanatory variable: $H G F_{i t}$ & $-187.468 * * *(13.251)$ & $-41.561 *(29.600)$ \\
\hline \multicolumn{3}{|l|}{ Control variables } \\
\hline \multicolumn{3}{|c|}{ Firm size dummy (Reference category: 25-49 employees) } \\
\hline $1-4$ & $16.729 * * *(0.689)$ & $8.363 * * *(0.802)$ \\
\hline $5-9$ & $13.559 * * *(0.684)$ & $7.139 * * *(0.789)$ \\
\hline $10-14$ & $10.557 * * *(0.697)$ & $5.490 * * *(0.839)$ \\
\hline $15-19$ & $8.326 * * *(0.788)$ & $4.435 * * *(0.917)$ \\
\hline $20-24$ & $5.347 * * *(0.910)$ & $3.843 * * *(1.064)$ \\
\hline $50-99$ & $-15.406 * * *(1.910)$ & $-4.795(2.964)$ \\
\hline $100-249$ & $-41.836^{* * *}(4.917)$ & $-29.717 * * *(5.561)$ \\
\hline $250-499$ & $-197.855 * * *(24.533)$ & $-48.961 * *(19.715)$ \\
\hline $500-1000$ & $-305.331 * * *(73.982)$ & $-125.530(98.184)$ \\
\hline Age & $-0.037(0.046)$ & no \\
\hline Industry size & $0.040(0.042)$ & $0.036 *(0.022)$ \\
\hline Industry growth & $0.205(0.177)$ & $0.136 * *(0.067)$ \\
\hline Industry code & yes & yes \\
\hline District & yes & yes \\
\hline Legal & yes & yes \\
\hline Constant & $-19.306^{* * *}(0.716)$ & $-8.855(0.925)^{* * *}$ \\
\hline Observations & 44,514 & 19,206 \\
\hline $\mathrm{R}^{2}$ & 0.643 & 0.163 \\
\hline
\end{tabular}

Dependent variable: Growth after high-growth period of exiting firms based on Eq. (2). Growth measure: absolute formula $=S_{\text {it }+\tau}-S_{\text {it }+\tau-}$ ${ }_{3}$, where $\mathrm{S}$ indicates size measured in number of employees. For relative growth, Eq. (2) equals -1 and is not estimated. Robust standard errors in parentheses. Coefficients for industry code, district, and legal are available upon request

$* p<0.1 ; * * p<0.05$; *** $p<0.01$ 


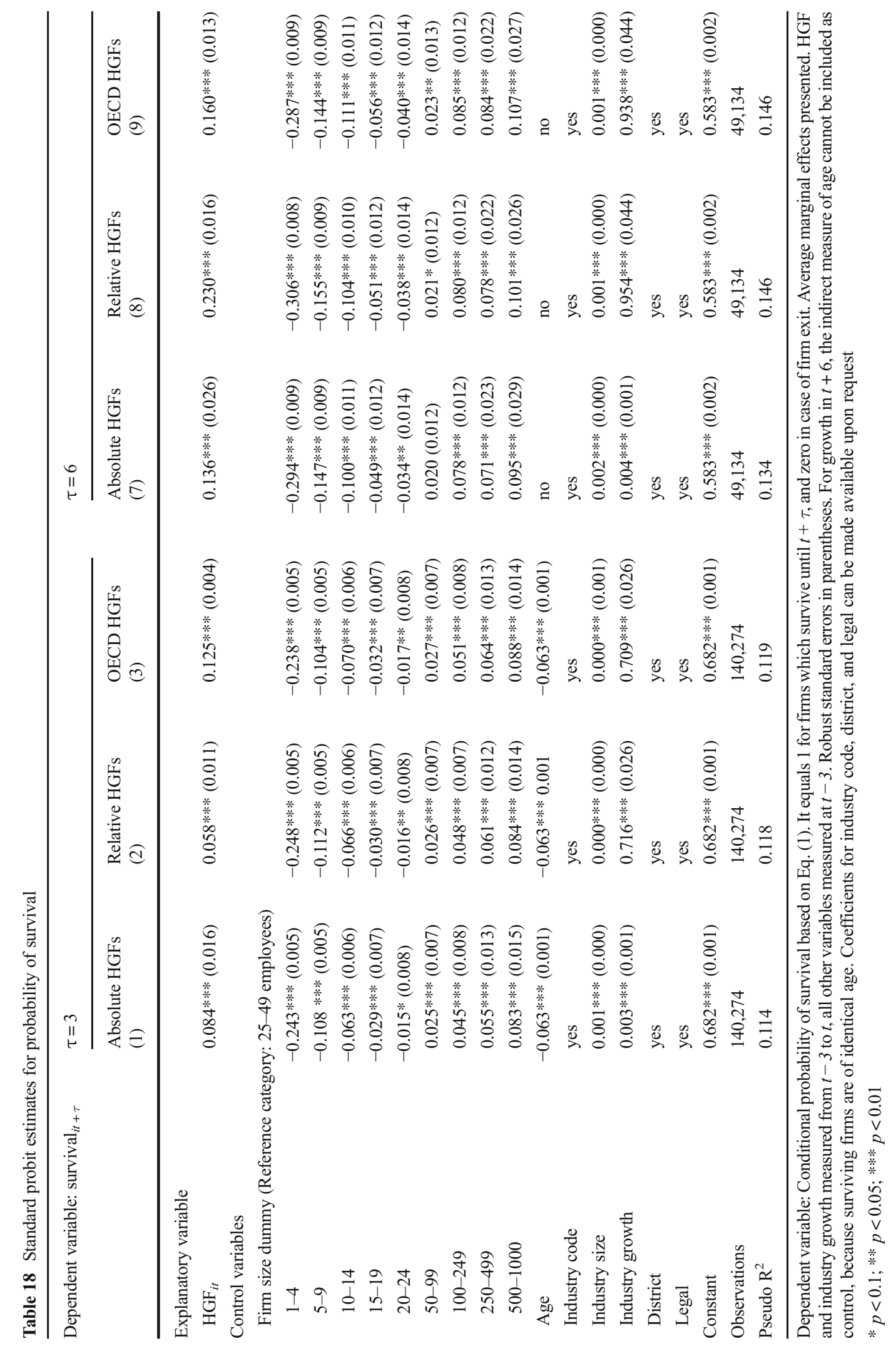

